\title{
Article
}

\section{Echo-Detected Electron Paramagnetic Resonance Spectra of Spin-Labeled Lipids in Membrane Model Systems}

Denis A. Erilov, Rosa Bartucci, Rita Guzzi, Derek Marsh, Sergei A. Dzuba, and Luigi Sportelli J. Phys. Chem. B, 2004, 108 (14), 4501-4507• DOI: 10.1021/jp037249y • Publication Date (Web): 16 March 2004

Downloaded from http://pubs.acs.org on March 24, 2009

\section{More About This Article}

Additional resources and features associated with this article are available within the HTML version:

- $\quad$ Supporting Information

- $\quad$ Links to the 1 articles that cite this article, as of the time of this article download

- Access to high resolution figures

- $\quad$ Links to articles and content related to this article

- Copyright permission to reproduce figures and/or text from this article

View the Full Text HTML

\section{ACS Publications}




\title{
Echo-Detected Electron Paramagnetic Resonance Spectra of Spin-Labeled Lipids in Membrane Model Systems
}

\author{
Denis A. Erilov, ${ }^{\dagger, l}$ Rosa Bartucci, ${ }^{*, \dagger}{ }^{\circ}$ Rita Guzzi, ${ }^{\dagger}$ Derek Marsh,, Sergei A. Dzuba, ${ }^{\ddagger}$ and \\ Luigi Sportelli ${ }^{\dagger}$ \\ Dipartimento di Fisica and Unità INFM, Università della Calabria, I-87036 Arcavacata di Rende (CS), Italy, \\ Abteilung Spektroskopie, Max-Planck-Institut fur biophysikalische Chemie, 37077 Gottingen, Germany, and \\ Institute of Chemical Kinetics and Combustion, Russian Academy of Science, \\ 630090 Novosibirsk, Russian Federation
}

Received: October 28, 2003; In Final Form: February 5, 2004

\begin{abstract}
The dynamics of spin-labeled lipid chains in the low-temperature phases of dipalmitoyl phosphatidylcholine (DPPC) membranes, with and without equimolar cholesterol, have been investigated by pulsed electron paramagnetic resonance (EPR) spectroscopy. Echo-detected spectra from the two-pulse, primary spin-echo (pulse sequence: $\pi / 2-\tau-\pi-\tau-$ echo) are used to detect rapid angular motions, on the time scale of the phase memory time $\left(T_{2 \mathrm{M}}\right)$ that is in the nanosecond regime. Echo-detected spectra from the three-pulse, stimulated spin-echo (pulse sequence: $\pi / 2-\tau-\tau / 2-T-\pi / 2-\tau-$ echo) are used to detect slow angular motions, on the time scale of the spin-lattice relaxation time $\left(T_{1}\right)$ that is in the microsecond regime. Spectra recorded at very low temperature $(77 \mathrm{~K})$ are used to correct the two-pulse echo spectra for instantaneous diffusion that arises from dipolar spin-spin interactions between different spin labels. Echo-detected inversion recovery spectra are used to correct the three-pulse echo spectra for intrinsic spin-lattice relaxation and large-scale spectral diffusion induced by nitrogen nuclear spin flips. The dependence of the echo-detected spectral line shapes on the two time delays, $\tau$ and $T$, can be simulated adequately by using a simple two-state model to represent the small-amplitude librational motions in the low-temperature membrane phases. The fast librational motion has isotropic character, no singly defined direction of the librational axis, and is segmental in nature, depending on chain position and also on the presence of cholesterol. The slow librational motion is of a more global, cooperative nature, being independent of chain position and cholesterol content.
\end{abstract}

\section{Introduction}

The dynamics of phospholipid chains in the low-temperature phases of bilayer membranes are relevant to the cryopreservation not only of membranes, but also of whole cells and tissues. In addition, molecular motions detected at low temperatures are inevitably present as part of the more complex membrane dynamical modes found at higher temperatures. Previously, we have shown that pulsed EPR of spin-labeled lipids at lowtemperature results in echo-detected (ED) spectra with characteristics that are very similar to those obtained from small spinlabel probes in glassy solvents. ${ }^{1}$ The latter have been investigated extensively, and it has been shown that the two-pulse ED-EPR spectral line shapes can be attributed to rapid, small-amplitude librations of the spin probe in glass-forming media. ${ }^{2-4}$

In the present work, we investigate, in greater detail, the motion of spin-labeled lipid chains in low-temperature phases of dipalmitoyl phosphatidylcholine membranes with and without $50 \mathrm{~mol} \%$ cholesterol. Special attention is paid to spectral simulation, and to the use of both two-pulse and three-pulse echo sequences. With the latter, it is possible to study slow angular motions on the microsecond time scale, ${ }^{5}$ in addition to the rapid nanosecond motions that are detected by the standard

\footnotetext{
* Corresponding author. E-mail: bartucci@fis.unical.it.

† Università della Calabria.

$\doteqdot$ Russian Academy of Science.

$\S$ Max-Planck-Institut fur biophysikalische Chemie.

" Present address: Department of Physics, Novosibirsk State University, 630090 Novosibirsk, Russian Federation.
}

two-pulse technique. ${ }^{6,7}$ It is found necessary to correct the twopulse spectra for instantaneous diffusion, and to correct the threepulse spectra for spin-lattice relaxation. Methods for doing this are developed and described here. Both slow and rapid librational motions are detected in membranes at low temperature. The fast motions depend on chain position and are influenced by the presence of cholesterol, whereas the slow motions are not. This suggests the presence of both segmental and wholebody librational motions of the lipid molecules in frozen membranes.

\section{Theoretical Background} by $^{8}$

The resonance frequency of a nitroxide spin label is given

$$
\omega_{\mathrm{m}}(\theta, \varphi)=\frac{g_{z z}(\theta, \varphi)}{g_{\mathrm{e}}} \gamma B+\gamma m a_{0}(\theta, \varphi)
$$

where $\gamma$ is the electron gyromagnetic ratio, $z$ is the axis of the laboratory frame directed along the magnetic field $\mathbf{B}$, the angles $\theta$ and $\varphi$ are the polar angles specifying the direction of $\mathbf{B}$ in the molecular frame, and $m$ is the projection of the nitrogen nuclear spin on its quantisation axis, which for ${ }^{14} \mathrm{~N}$ nuclei is equal to \pm 1 or 0 . The angular dependent $g$-value and hyperfine coupling constant are, respectively, 


$$
g_{z z}(\theta, \varphi)=g_{x x} \sin ^{2} \theta \cos ^{2} \varphi+g_{y y} \sin ^{2} \theta \sin ^{2} \varphi+g_{z z} \cos ^{2} \theta
$$

and

$$
\begin{array}{r}
a_{0}(\theta, \varphi)=\left(A_{x x}^{2} \sin ^{2} \theta \cos ^{2} \varphi+A_{y y}^{2} \sin ^{2} \theta \sin ^{2} \varphi+\right. \\
\left.A_{z z}^{2} \cos ^{2} \theta\right)^{1 / 2}
\end{array}
$$

where $g_{x x}, A_{x x}$, etc. are the principal values of the $\mathbf{g}$-tensor and of the hyperfine interaction tensor, respectively.

In ref 2 amd 3, a librational model was developed that assumes only small-amplitude reorientation takes place in rigid molecular glasses. For rotation around the nitroxide molecular $x$-axis that is of small amplitude $\alpha$, the change in resonance frequency, $\Delta \omega$, caused by the libration is

$$
\begin{aligned}
& \Delta \omega(\theta, \varphi)= \\
& \quad \alpha\left\{\frac{\gamma B}{g_{\mathrm{e}}}\left(g_{y y}-g_{z z}\right)+\frac{m \gamma\left(A_{y y}^{2}-A_{z z}^{2}\right)}{a_{0}(\theta, \varphi)}\right\} \sin \theta \cos \theta \sin \varphi
\end{aligned}
$$

For motion around the nitroxide $y$ - or $z$-axes, the principal values and the polar coordinates $\sin \theta \sin \varphi$ and $\cos \theta$ in eq 4 must be permuted appropriately.

The librational model ${ }^{2}$ is limited to the case in which motion is fast so that the Redfield limit, i.e., $\left\langle\Delta \omega^{2}\right\rangle \tau_{c}^{2} \ll 1$, is reached, where $\Delta \omega(t)$ is the stochastic fluctuation of the resonance frequency, the angular brackets indicate a time average, and $\tau_{\mathrm{c}}$ is the correlation time for motion. This theory predicts that relaxation is exponential, with a relaxation rate equal to $\left\langle\Delta \omega^{2}\right\rangle$ $\tau_{\mathrm{c} .}{ }^{9}$ It was shown in ref 3 , however, that also slow motion (i.e., $\left.\left\langle\Delta \omega^{2}\right\rangle \tau_{\mathrm{c}}^{2} \gg 1\right)$ occurs in simple organic glasses and manifests itself in the echo-detected EPR spectra. It is, therefore, desirable to employ a dynamic model that is applicable over the whole range of motional correlation times.

The simplest model of this kind is exchange between two equivalent sites. ${ }^{10}$ In analogy with the librational model, we assume that the two sites are related to a rotation around some particular axis in the molecular frame. Exchange between the orientations $\alpha=\alpha_{0}$ and $-\alpha_{0}$ results in exchange between two different resonance frequencies that are determined by eq 4 . This stochastic process results in transverse relaxation which influences the spin-echo decay.

For the two-pulse primary echo experiment (pulse sequence: $\pi / 2-\tau-\pi-\tau-$ echo) and the three-pulse stimulated echo (pulse sequence: $\pi / 2-\tau-\pi / 2-T-\pi / 2-\tau-$ echo), the decay of the echo amplitude is given by ${ }^{10}$

$$
\begin{aligned}
& E(2 \tau+T, \theta, \varphi)=\exp \left(-\frac{\tau}{\tau_{\mathrm{c}}}\right)\left[1+\frac{1}{2 \tau_{\mathrm{c}} R} \sinh 2 R \tau+\right. \\
& \left.\frac{1}{2 \tau_{\mathrm{c}}^{2} R^{2}} \sinh ^{2} R \tau-\frac{\Delta \omega^{2}(\theta, \varphi)}{R^{2}} \sinh ^{2} R \tau\left(1-\exp \left(-\frac{T}{\tau_{\mathrm{c}}}\right)\right)\right]
\end{aligned}
$$

where $\tau_{\mathrm{c}}$ is the correlation time (the exchange rate is: $\left.\left(2 \tau_{\mathrm{c}}\right)^{-1}\right)$, and $R^{2}=\left(2 \tau_{\mathrm{c}}\right)^{-2}-\Delta \omega^{2}(\theta, \varphi)$. Note that for the two-pulse experiment, $T=0$.

For fast motion, i.e., when $\Delta \omega^{2}(\theta, \varphi) \tau_{\mathrm{c}}^{2} \ll 1$, it follows from eq 5 that the echo decay is exponential:

$$
E(2 \tau+T, \theta, \varphi) \approx \exp \left(-2 \Delta \omega^{2}(\theta, \varphi) \tau_{\mathrm{c}} \tau\right)
$$

which conforms to the more general result that the decay due to fast molecular motion is independent of the motional mechanism. ${ }^{9}$ It follows from eqs 4 and 6 that, for fast motion, relaxation is determined by the parameter $\alpha_{0}^{2} \tau_{\mathrm{c}}$. In the opposite case of slow motion, i.e., when $\Delta \omega^{2}(\theta, \varphi) \tau_{\mathrm{c}}^{2} \gg 1$, one obtains the echo amplitude from eq 5 that is given by

$$
\begin{aligned}
E(2 \tau+T, \theta, \varphi)=\exp \left(-\frac{\tau}{\tau_{\mathrm{c}}}\right) \frac{1}{2}\left[1+\exp \left(-\frac{T}{\tau_{\mathrm{c}}}\right)+\right. \\
\left.\quad \cos (\Delta \omega(\theta, \varphi) \tau)\left(1-\exp \left(-\frac{T}{\tau_{\mathrm{c}}}\right)\right)\right]
\end{aligned}
$$

It is important to note that eq 6 does not depend on the time delay $T$ of the three-pulse stimulated echo experiment; i.e., this latter experiment is not sensitive to fast motion. ${ }^{5}$ This is a general property of the stimulated echo experiment because, for fast motion, the correlation between two phases-one acquired between the first and the second pulses, and the other acquired between the third pulse and the echo formation-is lost. On the other hand, eq 7 predicts that the two-pulse echo decay does not depend on $\theta$ and $\varphi$, so that the decay is isotropic and this experiment therefore is not sensitive to slow motion. Thus, a comparative study of ED-EPR spectra from the twopulse primary echo and three-pulse stimulated echo experiments allows conclusions to be reached about the time scale of motion and on its distribution between fast and slow components.

The echo-detected EPR line shape is obtained when the magnetic field $B$ is scanned across the resonance line. In the limit of small-amplitude motion, when $\Delta \omega(\theta, \varphi)$ is much less than the anisotropic broadening, the ED-EPR line shape is given by

$$
\begin{aligned}
& \mathrm{ED}^{\prime}(2 \tau+T, B)= \\
& \sum_{m} \frac{1}{4 \pi} \iint \sin \theta \mathrm{d} \theta \mathrm{d} \varphi f\left(B-\omega_{\mathrm{m}}(\theta, \varphi) / \gamma\right) E(2 \tau+T, \theta, \varphi)
\end{aligned}
$$

where $f(\Delta B)$ is the residual line broadening induced by unresolved hyperfine interactions with nuclei other than the spinlabel nitrogen. The function $f(\Delta B)$ is taken as a convolution of Gaussian and Lorentzian line shapes:

$$
\begin{aligned}
f(\Delta B)=\frac{1}{\delta_{\mathrm{L}} \delta_{\mathrm{G}} \sqrt{2 \pi}} \int \frac{1}{1+\left(\frac{\Delta B^{*}}{\delta_{\mathrm{L}}}\right)^{2}} \times \\
\quad \exp \left(-\frac{1}{2}\left(\frac{\left(\Delta B^{*}-\Delta B\right)}{\delta_{\mathrm{G}}}\right)^{2}\right) \mathrm{d}\left(\Delta B^{*}\right)
\end{aligned}
$$

where $\delta_{\mathrm{G}}$ is the Gaussian line width, and $\delta_{\mathrm{L}}$ is the Lorentzian line width.

An additional mechanism, which induces distortions in the line shape of the ED-EPR spectra, is the so-called instantaneous diffusion. For a uniform spatial distribution of spins, the decay law for this mechanism is ${ }^{11}$

$$
v(2 \tau, B, C)=\exp \left(-2 \tau \frac{4 \pi^{2}}{9 \sqrt{3}} \gamma^{2} \hbar C\left\langle\sin ^{2}(\xi / 2)\right\rangle\right)
$$

where $B$ is the spectrometer magnetic field, $C$ is the local spin concentration, $\xi$ is the turning angle of the magnetization by the second microwave pulse, and $\langle\ldots\rangle$ denotes averaging over the EPR spectrum. The factor $\left\langle\sin ^{2}(\xi / 2)\right\rangle$ reflects the fraction of spins that are excited by the microwave pulse. If the EPR line shape is described by the function $g(B)$, then 


$$
\begin{aligned}
& \left\langle\sin ^{2}(\xi / 2)\right\rangle= \\
& \frac{\int g\left(B^{\prime}\right) \mathrm{d} B^{\prime} \frac{B_{1}{ }^{2}}{\left(B^{\prime}-B\right)^{2}+B_{1}{ }^{2}} \sin ^{2}\left(\frac{\gamma t_{\mathrm{p}}}{2} \sqrt{\left(B^{\prime}-B\right)^{2}+B_{1}{ }^{2}}\right)}{\int g\left(B^{\prime}\right) \mathrm{d} B^{\prime}}
\end{aligned}
$$

where $B_{1}$ is the amplitude of the second pulse, and $t_{\mathrm{p}}$ is its duration. The resultant echo-detected line shape is finally given by the product

$$
\mathrm{ED}(2 \tau, B, C)=\mathrm{ED}^{\prime}(2 \tau, B) v(2 \tau, B, C)
$$

where the second factor on the right allows for instantaneous diffusion.

\section{Materials and Methods}

Materials. Synthetic 1,2-dipalmitoyl-sn-glycero-3-phosphocholine (DPPC) and cholesterol were obtained from Sigma/ Aldrich (St. Louis, MO). Spin-labeled phosphatidylcholine (1acyl-2-(n-doxyl)stearoyl-sn-glycero-3-phosphocholine) with $n$ $=5$ (5-PCSL) was obtained from Avanti Polar lipids (Birmingham, AL), whereas that with $n=14$ (14-PCSL) was synthesized according to Marsh and Watts. ${ }^{12}$ Reagent grade salts for the 10 $\mathrm{mM}$ phosphate buffer solution (PBS) at $\mathrm{pH}=7.5$ were from Merck (Darmstadt, Germany). All materials were used as purchased with no further purification. Distilled water was used throughout.

Sample Preparation. DPPC with $1 \mathrm{~mol} \%$ of either 5- or 14-PCSL, with and without $50 \mathrm{~mol} \%$ cholesterol, were codissolved in chloroform. The solvent was first evaporated with a nitrogen gas stream, and then residual traces of solvent were removed by drying under vacuum overnight. The lipids were dispersed in PBS at $\mathrm{pH} 7.5$ at a concentration of ca. $100 \mathrm{mg} /$ $\mathrm{mL}$ by vortex mixing with heating to $60{ }^{\circ} \mathrm{C}$, i.e., above the chain-melting phase transition of DPPC. The hydrated lipid bilayers were finally transferred to a standard $4 \mathrm{~mm}$ diameter, quartz EPR tube, concentrated by pelletting in a benchtop centrifuge, and the excess water was removed. Samples were incubated for $24 \mathrm{~h}$ at $10^{\circ} \mathrm{C}$ before measuring.

Pulsed EPR Spectroscopy. Two- and three-pulse, and inversion recovery, echo-detected EPR spectra were collected on a ELEXSYS E580 $9 \mathrm{GHz}$ Fourier Transform FT-EPR spectrometer (Bruker, Germany) equipped with a MD5 dielectric resonator and a CF 935P cryostat (Oxford Instruments, UK).

The ED-EPR spectra were obtained by recording the integrated spin-echo signal when sweeping the magnetic field. The integration window was $160 \mathrm{~ns}$. The microwave pulse widths were 32 and $64 \mathrm{~ns}$, with the microwave power adjusted to provide $\pi / 2$ - and $\pi$-pulses, respectively. The two-pulse sequence $(\pi / 2-\tau-\pi-\tau-$ echo $)$ and stimulated echo pulse sequence $(\pi /$ $2-\tau-\pi / 2-T-\pi / 2-\tau-$ echo) were used. For the inversionrecovery experiment $(\pi-T-\pi / 2-\tau-\pi-\tau-$ echo), the second and third pulses were the same as in the two-pulse experiment, and the first pulse was of $32 \mathrm{~ns}$ duration with its amplitude adjusted separately in the second pulse channel to provide a $\pi$-pulse. For the stimulated echo experiment, phase-cycling was used to cancel unwanted echoes that overlapped with the desired one at small $T$-delays.

\section{Results and Discussion}

Two-Pulse ED-EPR Spectra. Two-pulse ED-EPR spectra were collected for different values of the interpulse delay time, $\tau(216,352,488$, and $624 \mathrm{~ns})$. To exclude all field-independent

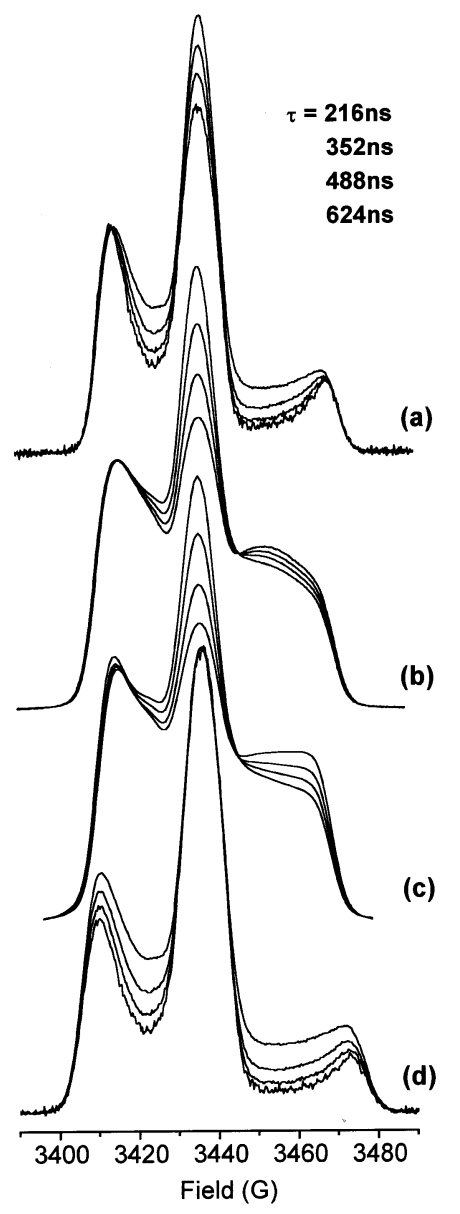

Figure 1. Two-pulse, echo-detected EPR spectra of 5-PCSL in bilayer membranes of DPPC $+50 \mathrm{~mol} \%$ cholesterol. (a) Original spectra at $200 \mathrm{~K}$ for different interpulse separations, $\tau$ (increasing from top to bottom). Spectra are normalized to the same intensity of the left shoulder. (b) Original spectra as in (a), except at 77 K. (c) Simulated spectra taking into account instantaneous diffusion according to eqs 10-12, with the parameters given in Table 1, and without motional relaxation. (d) Spectra of Figure 1a at $200 \mathrm{~K}$, corrected for instantaneous diffusion according to eq 13; spectra are normalized to the same intensity of the central line.

relaxation processes, the spectra were normalized to the same amplitude at some selected field position. Figure 1 shows results for the 5-PCSL spin label in cholesterol-containing DPPC bilayers that were recorded at temperatures of $200 \mathrm{~K}$ (Figure 1a) and at $77 \mathrm{~K}$ (Figure 1b).

One can see from Figure $1 \mathrm{~b}$ that at $77 \mathrm{~K}$ the amplitude of the central spectral component decreases with increasing $\tau$. Because motion is not expected for a lipid label in bilayer membranes at such a low temperature, this distortion of the line shape must be attributed to instantaneous diffusion. ${ }^{13}$ According to the theory, ${ }^{11}$ the decay rate arising from this mechanism is proportional to the fraction of spins excited by the microwave pulse, $\left\langle\sin ^{2}(\xi / 2)\right\rangle$ (see eq 10). This fraction attains its maximum value for the magnetic field positioned at the maximum of the central spectral component. Because the fraction of spins excited depends on the amplitude of the pulse, experiments were performed in which the amplitude $B_{1}$ of the second microwave pulse was varied while the same turning angle was maintained, to verify this mechanism. With decreasing amplitude of the pulse, the decrease in amplitude of the central spectral component was found to become much smaller (data not shown).

Simulation of the low-temperature spectra was performed by taking into account instantaneous diffusion according to eqs 10- 
TABLE 1: Spin Hamiltonian Tensors $\left(g_{I i}, A_{I i}\right)$ and Local Spin Concentrations $(C)$, and Linewidths $\left(\delta_{\mathrm{L}}, \delta_{\mathrm{G}}\right)$ and Amplitude-correlation Time Products $\left(\alpha_{0}^{2} \tau_{c}\right)$, Obtained from Simulation of Two-pulse ED-EPR Spectra at 77 and 200 K, Respectively

\begin{tabular}{lcccccccccc}
\hline \multicolumn{1}{c}{ sample/spin label } & $g_{x x}$ & $g_{y y}$ & $g_{z z}$ & $A_{x x}(\mathrm{G})$ & $A_{y y}(\mathrm{G})$ & $A_{z z}(\mathrm{G})$ & $C(\mathrm{mM})$ & $\delta_{\mathrm{L}}(\mathrm{G})$ & $\delta_{\mathrm{G}}(\mathrm{G})$ & $\alpha_{0}{ }^{2} \tau_{\mathrm{c}}\left(\mathrm{rad}^{2} \mathrm{~s}\right)$ \\
\hline DPPC/5-PCSL & 2.0089 & 2.0059 & 2.0024 & 4.0 & 3.5 & 33.8 & 1.6 & 0.6 & 2.1 & $5.5 \times 10^{-12}$ \\
DPPC/14-PCSL & 2.0089 & 2.0059 & 2.0024 & 4.1 & 3.9 & 31.7 & 1.3 & 0.5 & 2.0 & $6.5 \times 10^{-12}$ \\
DPPC+Chol/5-PCSL & 2.0089 & 2.0059 & 2.0024 & 4.2 & 3.6 & 34.6 & 2.6 & 0.6 & 2.0 & $5.0 \times 10^{-12}$ \\
DPPC+Chol/14-PCSL & 2.0089 & 2.0059 & 2.0024 & 4.2 & 3.8 & 32.0 & 2.5 & 0.5 & 2.0 & $8.7 \times 10^{-12}$
\end{tabular}

12. The results of these calculations are given in Figure 1c. It can be seen that this mechanism describes the experimental spectra in Figure 1b rather well, and that the value of the local spin-label concentration, $C$, can be obtained from the simulations. The simulation parameters, including effective local concentrations, are given in Table 1. For comparison, the effective concentration of the lipid molecules in a bilayer membrane is in the region of $1 \mathrm{M}$.

The effective local spin concentrations, $C$, derived from the effect of instantaneous diffusion are higher in membranes that contain $50 \mathrm{~mol} \%$ cholesterol than in those of DPPC alone, although the spin label/(total lipid) molar ratio is the same in all samples. On the other hand, it was deduced from the line widths of the conventional EPR spectra that the local spin concentration is substantially higher in samples without cholesterol than in those containing cholesterol. ${ }^{1}$ The additional line broadening in samples without cholesterol amounts to $2-3 \mathrm{G}$, which is equivalent to transverse relaxation times of 200-100 $\mathrm{ns}$, respectively. Because the first measurement is made already with a delay time $2 \tau=432 \mathrm{~ns}$, these dipolar-broadened spins are not detected in the echo experiment. This prediction is confirmed by the fact that the echo signal in samples with DPPC alone is approximately 10 times smaller than in the samples containing cholesterol. The residual spins that are detected in the echo from samples containing DPPC alone are consequently more dilute than those in cholesterol-containing membranes. In the latter case, the spins are more uniformly distributed in the membrane, rather than being preferentially squeezed out from the host lipid, as in DPPC alone.

Because the instantaneous diffusion is essentially independent of temperature, we expect its influence to be the same at $200 \mathrm{~K}$ as that observed in the absence of motion at $77 \mathrm{~K}$. This is true to the extent that the effective local spin-label concentration does not change. With this assumption, it is possible to correct the spectra for the effects of instantaneous diffusion at $200 \mathrm{~K}$ by using the data from $77 \mathrm{~K}$, together with the multiplicative relation given in eq 12 . Neglecting any changes in the magneticresonance parameters over this temperature range, the corrected spectra at $200 \mathrm{~K}$ are given by

$$
\mathrm{ED}_{200 \mathrm{~K}}^{*}(B, \tau)=\mathrm{ED}_{200 \mathrm{~K}}(B, \tau) \frac{\mathrm{ED}_{77 \mathrm{~K}}\left(B, \tau_{1}\right)}{\operatorname{ED}_{77 \mathrm{~K}}(B, \tau)}
$$

where $\mathrm{ED}_{T}(\mathrm{~B}, \tau)$ is the ED-EPR line shape at temperature $T$ and time delay $\tau$, the asterisk denotes the corrected spectrum, and $\tau_{1}$ is the shortest value of $\tau$ used in the experimental series. After this correction, the spectra still contain residual effects of instantaneous diffusion, because a vanishingly small value of $\tau_{1}$ cannot be realized experimentally. However, the resulting distortion is unlikely to be large and it is constant for the different values of $\tau$. Moreover, it can easily be taken into account in spectral simulation (see below).

The corrected ED-EPR spectra (i.e., substantially free of effects from instantaneous diffusion) are shown in Figure 1d, for 5-PCSL in cholesterol-containing DPPC bilayers at $200 \mathrm{~K}$. One can see a decrease in amplitude in the low-field and high-
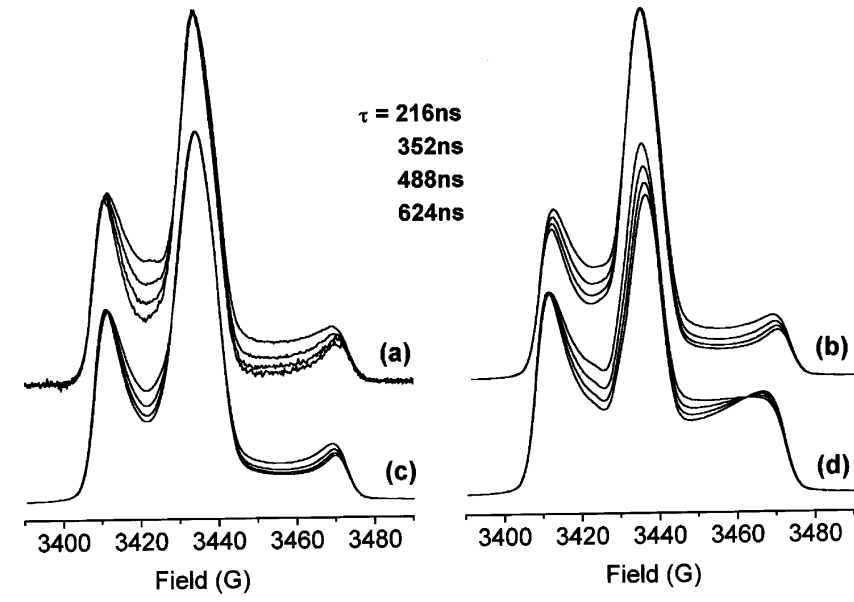

Figure 2. (a) Two-pulse, echo-detected EPR spectra of 14-PCSL in DPPC bilayer membranes at $200 \mathrm{~K}$. Spectra are recorded for different interpulse delays, $\tau$ (increasing from top to bottom), are corrected for instantaneous diffusion, and are normalized to the central lineheight. (b) Two-pulse spectra simulated for librational motion about the nitroxide $x$-molecular axis, according to eqs 4,6 , and 8 with $\alpha_{0}^{2} \tau_{\mathrm{c}}=$ $1.3 \times 10^{-11} \mathrm{rad}^{2} \mathrm{~s}$. (c) Spectra simulated for librational motion about the nitroxide $y$-molecular axis, with $\alpha_{0}^{2} \tau_{\mathrm{c}}=2.8 \times 10^{-11} \mathrm{rad}^{2} \mathrm{~s}$. (d) Spectra simulated for librational motion about the nitroxide $z$-molecular axis, with $\alpha_{0}^{2} \tau_{\mathrm{c}}=2.8 \times 10^{-10} \mathrm{rad}^{2} \mathrm{~s}$. Other simulation parameters are given in Table 1 .

field wings of the spectrum, on increasing the interpulse spacing, $\tau$. Near the canonical orientations, the decrease is smaller than at the intermediate positions. These features are typical of smallamplitude, restricted reorientational motion, for which the magnetization at the canonical nitroxide orientations relaxes more slowly than at the intermediate orientations. ${ }^{2}$ This type of motion has been detected previously by using pulsed EPR of small nitroxides in molecular glasses. ${ }^{2-4}$ For these latter cases, it was shown that a model of fast, small-amplitude reorientational motion provides good quantitative agreement with experiment. ${ }^{3}$

Simulation of Two-Pulse ED-EPR Spectra. To derive principal values of the magnetic tensors, and also the local spin concentrations that characterize the instantaneous diffusion, simulation was performed of spectra recorded at $77 \mathrm{~K}$, where it is assumed that the spin-labeled lipids are totally immobilized. The parameters obtained from these simulations are listed in Table 1. These values were then used in simulations of the spectra recorded at $200 \mathrm{~K}$. The individual line widths $\left(\delta_{\mathrm{G}}\right.$ and $\delta_{\mathrm{L}}$ ) were found by fitting the outer wings of the spectra at 200 $\mathrm{K}$.

In the limit of fast librational motion, simulation was performed for different orientations of the librational axis, viz., along the $x$-, $y$-, and $z$-molecular axes of the nitroxide. The simulation results are presented in Figure 2 for 14-PCSL in a DPPC:Chol 1:1 mol/mol mixture. As can be seen from comparison of Figure $2 \mathrm{a}, \mathrm{d}$, librational motion solely around the $z$-axis does not describe the "shoulders" of the experimental spectra even qualitatively. With fully extended chains, $z$ corresponds to the long axis of the lipid molecule that is directed 


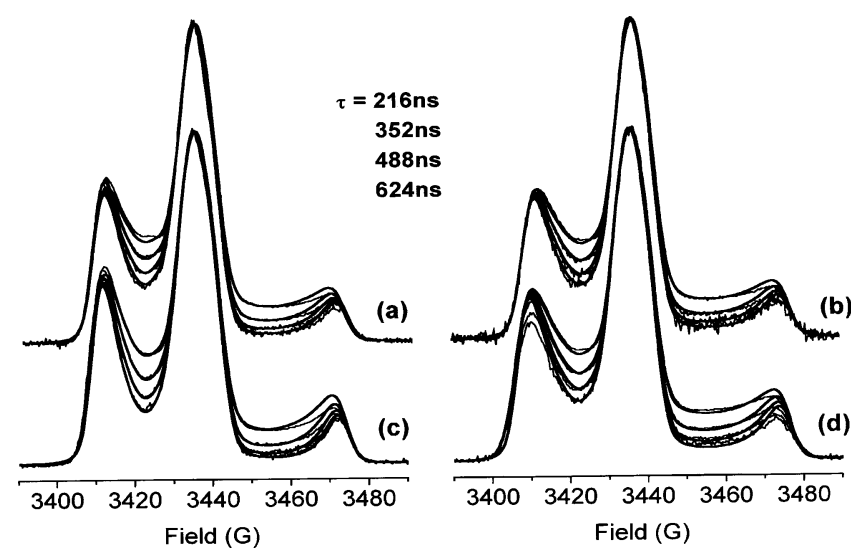

Figure 3. Two-pulse, echo-detected EPR spectra recorded at $200 \mathrm{~K}$ for different interpulse separations, $\tau$ (thin noisy lines), with spectra simulated for isotropic librational motion (thick lines). (a) 14-PCSL in DPPC bilayer membranes. (b) 5-PCSL in DPPC bilayer membranes. (c) 14-PCSL in bilayer membranes of DPPC $+50 \mathrm{~mol} \%$ cholesterol. (d) 5-PCSL in bilayer membranes of DPPC $+50 \mathrm{~mol} \%$ cholesterol. All spectra are normalized to the same intensity of the central line. Parameters used for the simulations are given in Table 1 .

preferentially along the membrane normal (see, e.g., ref 14). For reorientation around the $x$ - and $y$-axes (Figure 2b,c, respectively), which are orthogonal to the chain long-axis, the agreement is considerably better than that for rotation around the $\mathrm{z}$-axis but is still not entirely satisfactory.

A further model used for the simulations was the isotropic one. This model assumes that the librational axis adopts stochastically different directions at different moments of time. We describe this motion as simultaneous, independent (i.e., uncorrelated) librational motions around three perpendicular axes: $x, y$, and $z$.The net relaxation is given by the product of the three independent relaxations induced by each librational mode. This model gives an almost quantitative description of the ED-EPR spectra at $200 \mathrm{~K}$ for all samples studied (see Figure 3 ). Note that only a single fitting parameter is used in the simulation: the product of the angular amplitude and correlation time, $\alpha_{0}^{2} \tau_{\mathrm{c}}$. Values of this dynamic parameter that are obtained from simulation of the dependence of the ED-EPR line shapes on interpulse spacing (see Figure 3) are presented in the final column of Table 1 . The reason that the two-pulse ED-EPR spectra are better simulated by isotropic motions around three orthogonal axes is probably that the fast librations arise from segmental motions. Because the latter take place around all (noncollinear) $\mathrm{C}-\mathrm{C}$ bonds in the lipid molecule, the motion of the label at a given chain position is the sum of the reorientations about all preceding $\mathrm{C}-\mathrm{C}$ bonds.

The motional parameter $\alpha_{0}^{2} \tau_{\mathrm{c}}$ is known to increase with increasing temperature for small spin labels in molecular glasses. ${ }^{3,4,15}$ Larger values of this parameter therefore imply a greater intensity of motion. For membranes containing cholesterol, one can see from Table 1 that this parameter is larger at the terminal methyl end of the lipid chain (viz., 14-PCSL) than it is for a chain position close to the lipid polar headgroup (viz., 5-PCSL). This means that the terminal methyl end of the chain moves more freely. This increase in motional freedom can be attributed to the irregular shape of the cholesterol molecule: 5-PCSL is located adjacent to the rigid steroid nucleus, whereas 14-PCSL is situated in a void region beneath the cholesterol aliphatic tail (see, e.g., ref 16). In the absence of cholesterol, the DPPC chains are packed more regularly and the terminal methyl end is therefore more restricted.

Although, for fast motion, we can only obtain the amplitudecorrelation time product, $\alpha_{0}{ }^{2} \tau_{\mathrm{c}}$, some estimate of limits on $\tau_{\mathrm{c}}$ is
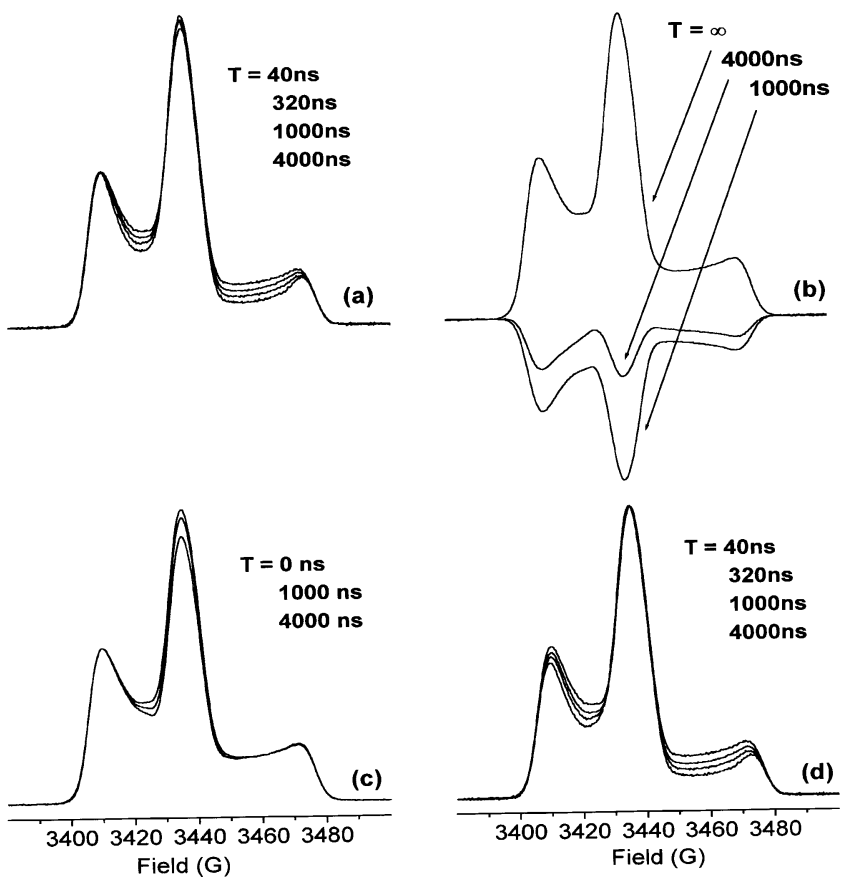

Figure 4. Three-pulse, stimulated echo-detected spectra of 5-PCSL in bilayer membranes of DPPC $+50 \mathrm{~mol} \%$ cholesterol at $200 \mathrm{~K}$, and their correction for longitudinal relaxation by using the inversionrecovery experiment. (a) Original three-pulse ED-EPR spectra recorded with $\tau=216 \mathrm{~ns}$ and different different values of the $T$-delay (increasing from top to bottom). Spectra are normalized to the same intensity of the left shoulder. (b) Echo-detected inversion-recovery spectra also recorded at $200 \mathrm{~K}$, with $\tau=216 \mathrm{~ns}$ and different values of the $T$-delay. (c) Inversion recovery data replotted according to eq 14 , and normalized to the intensity of the left shoulder. (d) Three-pulse ED-EPR spectra corrected according to eq 15 , by using the data from (c). Spectra are normalized to the height of the center line.

possible. For 14-PCSL in DPPC bilayer membranes we obtain $\alpha_{0}^{2} \tau_{\mathrm{c}}$ equal to $6.5 \times 10^{-12} \mathrm{rad}^{2} \mathrm{~s}$ (see Table 1 ), which gives $\Delta \omega^{2} \tau_{\mathrm{c}}^{2} \approx 2 \times 10^{6} \mathrm{~Hz} \mathrm{rad}^{2}$, for the center of the high-field hyperfine component. Because $\Delta \omega^{2} \tau_{\mathrm{c}}{ }^{2} \ll 1$ for fast motion, this gives $\tau_{\mathrm{c}} \ll 5 \times 10^{-7} \mathrm{~s}$. We may also assume that $\alpha_{0}<0.2 \mathrm{rad}$; otherwise line narrowing must be observed between 77 and 200 $\mathrm{K}$, which is not the case. From this it follows that $\tau_{\mathrm{c}}>10^{-9} \mathrm{~s}$. So we find that the correlation time for the librational motion is on the nanosecond scale and restricted to the range $10^{-9}<$ $\tau_{\mathrm{c}} \ll 5 \times 10^{-7} \mathrm{~s}$.

Three-Pulse Stimulated and Inversion Recovery ED-EPR Spectra. Figure 4a shows three-pulse ED-EPR spectra of 5-PCSL in DPPC bilayer membranes containing $50 \mathrm{~mol} \%$ cholesterol. Besides spectral diffusion, spin-lattice relaxation and large-scale spectral diffusion induced by nitrogen nuclear spin flips also contribute to the decay of the stimulated echo. These latter contributions can be determined in an independent experiment by using the inversion-recovery (IR) technique, with a pulse sequence $\pi-T-\pi / 2-\tau-\pi-\tau-$ echo, where the time delay $T$ is varied and $\tau$ is fixed. Echo-detected inversionrecovery spectra for the same sample as in Figure $4 \mathrm{a}$ are presented in Figure 4b. (The spectrum for $T=\infty$ was recorded with the first $\pi$-pulse switched off.) In Figure $4 c$, the inversion recovery spectra are replotted according to the equation

$$
\mathrm{ED}_{\mathrm{IR}}^{*}(B, T)=\mathrm{ED}_{\mathrm{IR}}(B, \infty)-\mathrm{ED}_{\mathrm{IR}}(B, T)
$$

where $\operatorname{ED}_{\mathrm{IR}}(B, T)$ is the intensity of the inversion-recovery EDEPR spectrum at field position $B$ and time delay $T$. For $T=0$, we used: $\operatorname{ED}_{\mathrm{IR}}(B, 0)=-\operatorname{ED}_{\mathrm{IR}}(B, \infty)$. The spectra in Figure $4 \mathrm{c}$ 


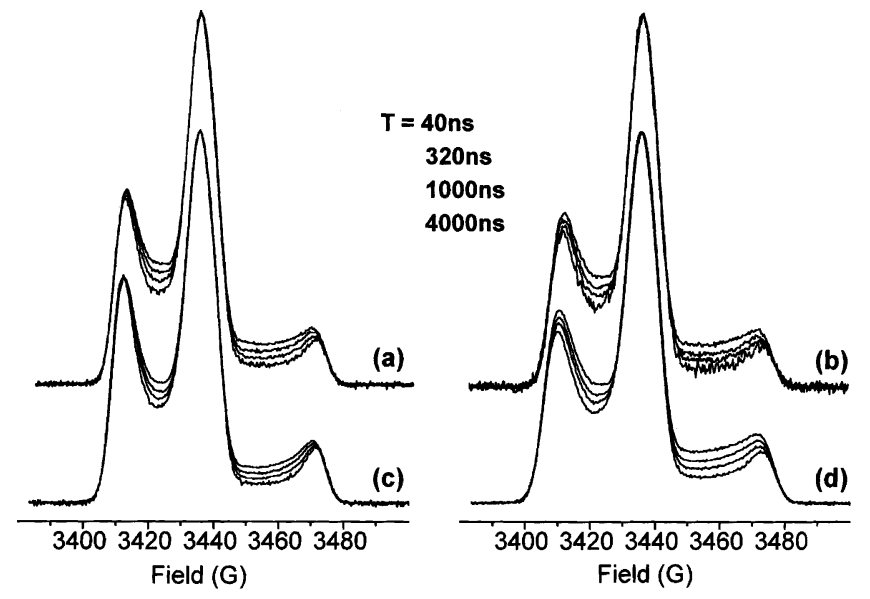

Figure 5. Three-pulse, stimulated-echo ED-EPR spectra of $n$-PCSL spin labels in lipid bilayer membranes at $200 \mathrm{~K}$. Spectra are recorded with $\tau=216 \mathrm{~ns}$ and different values of the $T$-delay (increasing from top to bottom) and are corrected for spin-lattice relaxation. (a) 14PCSL in DPPC bilayers. (b) 5-PCSL in DPPC bilayers. (c) 14-PCSL in bilayers of DPPC $+50 \mathrm{~mol} \%$ cholesterol. (d) 5-PCSL in bilayers of DPPC $+50 \mathrm{~mol} \%$ cholesterol (identical to Figure $4 \mathrm{~d}$ ).

are normalized to the same low-field peak height. Recalculated in this way, the inversion-recovery spectra may be compared directly with the stimulated echo spectra that are given in Figure 4a. Then, to obtain the contribution from spectral diffusion, the stimulated ED-EPR spectra can be corrected for spin-lattice relaxation in the following way:

$$
\mathrm{ED}_{\mathrm{SE}}^{*}(B, T)=\mathrm{ED}_{\mathrm{SE}}(B, T) \frac{\mathrm{ED}_{\mathrm{IR}}^{*}(B, 0)}{\operatorname{ED}_{\mathrm{IR}}^{*}(B, T)}
$$

where the asterisk on the left-hand side denotes the corrected stimulated ED-EPR spectrum. This procedure is similar to the correction of the primary ED-EPR spectra for instantaneous diffusion that was given above. The result is shown in Figure $4 \mathrm{~d}$. As it was not possible to obtain inversion-recovery spectra at short $T$-delays (40 ns, $320 \mathrm{~ns}$ ), because of overlap with unwanted echoes, inversion-recovery ED-EPR spectra necessary for correction of the stimulated ED-EPR spectra at $T=40$ ns and $320 \mathrm{~ns}$ were obtained by interpolation between spectra at $T=0$ and $1000 \mathrm{~ns}$, assuming that the inversion recovery is exponential. (It was not possible to apply phase-cycling in the inversion recovery experiment because of the limited number of pulse channels). The effective spin-lattice relaxation times that were obtained from inversion recovery experiments were on the microsecond time scale, the same order of magnitude of the values found for the n-PCSL spin labels in gel phase DPPC membranes. ${ }^{17,18}$

Simulation of Three-Pulse ED-EPR Spectra. Figure 5 presents the experimental stimulated-echo ED-EPR spectra that are corrected for $T_{1}$ relaxation by using the inversion recovery experiments. Corrected spectra are given for both positions of spin labeling, and for membranes with and without cholesterol. Simulations of the stimulated-echo ED-EPR spectra by using the two-site model (i.e., eq 5) with isotropic character (see above) are shown in Figure 6 for 14-PCSL in DPPC bilayer membranes. One can see that good qualitative agreement is obtained with the experimental spectra. The parameters used for the simulations are $\tau_{\mathrm{c}} *=0.8 \mu \mathrm{s}$ and $\alpha_{0} *=1.7 \times 10^{-2} \mathrm{rad}$, where the asterisk indicates parameters that are associated with slow motion. These parameters must be viewed as approximate estimates because only qualitative agreement was achieved

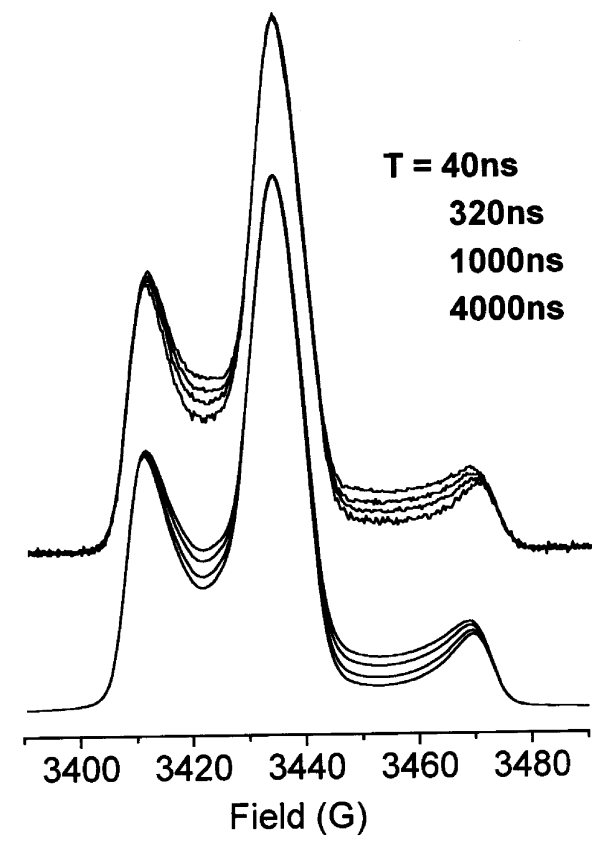

Figure 6. Top: three-pulse, stimulated-echo detected spectra of 14PCSL in DPPC bilayer membranes at $200 \mathrm{~K}$. Spectra are recorded with $\tau=216 \mathrm{~ns}$ and different values of the $T$-delay (increasing from top to bottom) and are corrected for spin-lattice relaxation. Bottom: spectra simulated with the two-site model according to eqs 5 and 8. Parameters used in the simulation are $\tau_{\mathrm{c}}{ }^{*}=0.8 \mu \mathrm{s}$ and $\alpha_{0} *=1.7 \times 10^{-2} \mathrm{rad}$, and otherwise as in Table 1 (row 2).

between simulated and experimental ED spectra. For a maximal spectral extent $\Delta \omega^{*}$, this latter correlation time yields a value of $\Delta \omega^{* 2} \tau_{\mathrm{c}}^{* 2} \approx 35$, for the high-field hyperfine manifold, i.e., $\Delta \omega^{* 2} \tau_{\mathrm{c}}^{* 2} \gg 1$, which confirms that indeed slow motion is detected in the stimulated echo experiment. It also follows from this result that the amplitude-correlation time product is $\alpha_{\mathrm{o}}^{* 2} \tau_{\mathrm{c}}^{*}$ $=2.3 \times 10^{-10} \mathrm{rad}^{2} \mathrm{~s}$, which is almost 1 order of the magnitude greater than that found from the two-pulse experiment (cf. Table 1). Thus a wide distribution of correlation times exists for the librational motions of the lipid chains in DPPC membranes, with and without cholesterol, at $200 \mathrm{~K}$. This distribution extends at least up to the microsecond time scale, as illustrated by the results from the three-pulse experiments.

According to eq 5, the dependence of the stimulated echo on delay time $T$ is exponential, in the two-site model, with a characteristic time that is of the order of the correlation time for the motion. In Figure 7, we plot the $T$-dependence of the spectral amplitude at the center of the high-field manifold $(B$ $=3455 \mathrm{G}$ ), for 5-PCSL in membranes of DPPC $+50 \mathrm{~mol} \%$ cholesterol at $200 \mathrm{~K}$ (i.e., for Figure 5d). This corresponds to the spectral position that is most sensitive to librational dynamics. Exponential fitting shown in Figure 7 yields a decay time $\tau_{\mathrm{c}}^{*}=0.78 \pm 0.11 \mu \mathrm{s}$. Following this method, the correlation time of the slow motion was obtained for all samples (see Table 2). The value obtained for 14-PCSL in membranes of DPPC alone agrees rather well with that obtained above from qualitative spectral simulation. Note that the correlation time $\tau_{\mathrm{c}}{ }^{*}$ of the slow motional component is rather similar for the different samples studied. This differs from the situation for the fast motional component that is observed in the two-pulse experiment. In the latter case, the product $\alpha_{0}^{2} \tau_{\mathrm{c}}$ is greater for 14-PCSL than for 5-PCSL, especially in the presence of cholesterol. Most probably, the fast libration represents local segmental motion of the lipid chains, whereas the slow motion reflects cooperative motions of the entire lipid molecule. 


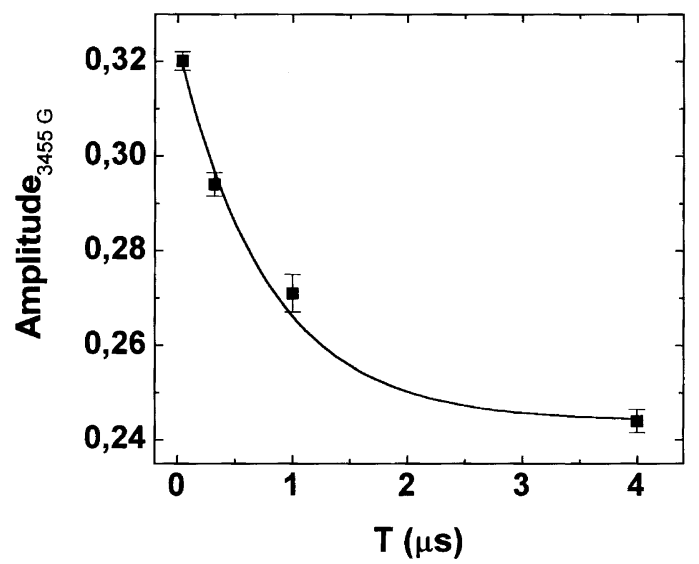

Figure 7. Amplitude at $3455 \mathrm{G}$ field position of three-pulse ED-EPR spectra from 5-PCSL in DPPC $+50 \mathrm{~mol} \%$ cholesterol at $200 \mathrm{~K}$ (i.e., Figure 5d), as a function of the $T$-delay. The solid line is an exponential least-squares fit to the data according to eq 7 . The decay time of the exponential is $0.78 \pm 0.11 \mu \mathrm{s}$.

TABLE 2: Correlation Times $\left(\tau_{\mathrm{c}}^{*}\right)$ for the Slow Motion Obtained from the Exponential Fitting of the Three-Pulse ED-EPR Spectra at $200 \mathrm{~K}$

\begin{tabular}{lc}
\hline sample/spin label & $\tau_{\mathrm{c}}^{*}(\mu \mathrm{s})$ \\
\hline DPPC/5-PCSL & $0.81 \pm 0.35$ \\
DPPC/14-PCSL & $0.82 \pm 0.22$ \\
DPPC +Chol/5-PCSL & $0.78 \pm 0.11$ \\
DPPC +Chol/14-PCSL & $0.91 \pm 0.25$
\end{tabular}

\section{Conclusions}

In this study of lipid chain dynamics in the low-temperature phases of spin-labeled membranes, a good quantitative agreement between theory and experiment is obtained for the conventional, two-pulse, echo-detected EPR experiment by using the model of fast librational motion. Because this experiment is sensitive to instantaneous diffusion, as well as to spin-label dynamics, we propose a means to correct for spectral distortions that are induced by the former mechanism. The correction requires spectra to be obtained at sufficiently low temperature that all motion is frozen out.

Our comparative study of two-pulse and three-pulse ED-EPR spectra reveals the presence of a wide distribution of correlation times for the librational chain motions in the low-temperature phases of lipid membranes. This approach exploits the fact that the two kinds of experiment are sensitive to quite different time scales of molecular motion. We found that small-amplitude librations are present with correlation times in both the nanosecond and microsecond ranges, for all membrane states investigated. To simulate spectral changes in both the fast $\left(\Delta \omega^{2} \tau_{\mathrm{c}}^{2} \ll 1\right)$ and slow $\left(\Delta \omega^{2} \tau_{\mathrm{c}}^{2} \gg 1\right)$ motional regimes, a simple two-site model can be employed effectively. In this model, librational motion is represented as exchange between two orientations, fixed in angular space. With the two-pulse EDEPR experiment, the composite amplitude-correlation time parameter, $\alpha_{0}^{2} \tau_{\mathrm{c}}$, for the fast motion can be determined. By using the three-pulse, stimulated echo experiment, both the correlation time, $\tau_{\mathrm{c}}{ }^{*}$, and amplitude, $\alpha_{0}{ }^{*}$, for the slow motional component can be obtained separately.

An increase in the fast motional parameter $\alpha_{0}^{2} \tau_{\mathrm{c}}$ is found on going from the polar headgroup end of the lipid chain (viz., 5-PCSL) to its free, terminal-methyl end (viz., 14-PCSL). It is known that $\alpha_{0}^{2} \tau_{\mathrm{c}}$ increases with increasing temperature for small spin labels in glassy media. ${ }^{4}$ Therefore, the present results are attributed to a gradient of increasing of librational freedom for fast segmental motion on proceeding down the lipid chains toward the terminal methyl group. This gradient is accentuated by the presence of cholesterol for reasons of molecular packing. On the other hand, the slow librations detected in the threepulse stimulated-echo experiment appear to be cooperative, whole-body motions that depend neither on chain-segment position nor on the presence of cholesterol.

Acknowledgment. We thank Frau B. Angerstein for synthesis of spin-labeled phospholipids. R.B., L.S., and D.M. are members of the COST D22 Action of the European Union. D.A.E. and S.A.D. thank INFM and CRDF (NO-008-X1) for financial support. This work was supported by a grant from the project CIPE-Cluster MIA26-WP3.

\section{References and Notes}

(1) Bartucci, R.; Guzzi, R.; Marsh, D.; Sportelli, L. J. Magn. Reson. 2003, 162, 371

(2) Dzuba, S. A.; Tsvetkov, Yu. D.; Maryasov, A. G. Chem. Phys. Lett. 1992, 188, 217.

(3) Kirilina, E. P.; Dzuba, S. A.; Maryasov, A. G.; Tsvetkov, Yu. D. Appl. Magn. Res. 2001, 21, 203.

(4) Dzuba, S. A. Spectrochim. Acta Part A 2000, 56, 227.

(5) Schwartz, L. J.; Millhauser, G. L.; Freed, J. H. Chem. Phys. Lett. 1986, 127, 60 .

(6) Millhauser, G. L.; Freed, J. H. J. Chem. Phys. 1984, 81, 37.

(7) Saxena, S.; Freed, J. H. J. Phys. Chem. A 1997, 101, 7998.

(8) Libertini, L. J.; Griffith, O. H. J. Chem. Phys. 1970, 53, 1359.

(9) Schlichter, C. P. Principles of Magnetic Resonance; Springer: Berlin, 1996.

(10) Zhidomirov, G. M.; Salikhov, K. M. Sov. Phys. JETP 1969, 29, 1037.

(11) Salikhov K. M.; Tsvetkov, Yu. D. In Time Domain Electron Spin Resonance; Kevan, L., Schwartz, R. N., Eds.; Wiley-Interscience: New York, 1979; pp 231-278.

(12) Marsh, D.; Watts, A. In Lipid-Protein Interactions; Jost, P. C., Griffith, O. H., Eds.; Wiley-Interscience: New York, 1982; Vol. 2, pp $53-$ 126.

(13) Toropov, Yu. V.; Dzuba, S. A.; Tsvetkov, Yu. D.; Monaco, V.; Formaggio, F.; Crisma, M.; Toniolo, C.; Raap, J. Appl. Magn. Reson. 1998, $15,237$.

(14) Gaffney, B. J.; Marsh, D. Proc. Natl. Acad. Sci. U.S.A. 1998, 95, 12940.

(15) Dzuba, S. A.; Watari, H.; Shimoyama, Y.; Maryasov, A. G.; Kodera, Yo.; Kawamori, A. J. Magn. Reson. A 1995, 115, 80.

(16) Kurad, D.; Jeschke, G.; Marsh, D. Appl. Magn. Reson. 2001, 21 , 469.

(17) Marsh, D.; Pali, T.; Horvath, L. I. In Spin Labeling: the Next Millenium Biological Magnetic Resonance; Berliner, L. J., Ed.; Plenum Press: New York, 1988; Vol 14, pp 23-82.

(18) Fajer, P.; Watts, A.; Marsh, D. Biophys. J. 1992, 61, 879. 